\title{
Cardiac rehabilitation: impact of graded exercise in the recovery period following myocardial infarction
}

This article was published in the following Dove Press journal:

Research Reports in Clinical Cardiology

25 July 2013

Number of times this article has been viewed

\author{
Simon White \\ School of Pharmacy, Keele University, \\ Staffordshire, UK
}

\begin{abstract}
This paper reviews the impact of graded exercise undertaken as part of a cardiac rehabilitation (CR) program in the recovery period following a myocardial infarction, focusing on how CR may be best provided and the evidence-base relating to exercise-based CR. Essential components of $\mathrm{CR}$ are considered here to include education about healthy behavior, lifestyle modification where necessary (especially in relation to smoking, diet, and physical exercise), medical risk factor management, use of cardioprotective medicines and implantable devices, and psychosocial health management. It is argued that the totality of the evidence continues to demonstrate benefits of exercise-based CR in terms of mortality and morbidity, despite the debate about the magnitude of that benefit. However, given the wide variance in the quality and nature of CR service provision, there is no guarantee that patients eligible for CR will benefit fully. In line with national and international standards, CR should be tailored to the patient's individual needs, but structured exercise is recommended for most patients. Exercise sessions, whether based in hospital, in the community, or at home, should be designed to vary the frequency, intensity, duration, and type of exercise. They must include an initial warm-up period, before a conditioning period, and finish with a cool-down period. Patients should be taught to self-monitor so that they can exercise safely on their own. In designing interventions to support patients to change health behavior, health professionals should recognize that patients may only make lifestyle modifications to aspects of lifestyle perceived as causes of their cardiovascular disease and so, for example, may not do the recommended amount of exercise if they do not perceive lack of exercise to be a cause of their cardiovascular disease.
\end{abstract}

Keywords: cardiovascular disease, public health, education programs, cardiac rehabilitation, lifestyle change, physical activity

\section{Introduction}

The mortality and morbidity burden of cardiovascular disease (CVD) has fallen substantially in most Westernized countries over the past 15 years. ${ }^{1}$ There are various reasons for this, including more effective drug therapy, increasing availability of effective cardiac surgical interventions, particularly percutaneous techniques, and primary and secondary prevention strategies. ${ }^{1,2}$ Cardiac rehabilitation $(\mathrm{CR})$ is a multidisciplinary secondary prevention strategy that focuses on patients' physical and psychological health and involves various interventions tailored to patients' needs, including graded and structured exercise. ${ }^{2}$ This is expressed in the following definition of CR:

$[\ldots]$ sum of activities required to influence $[\ldots]$ the underlying cause of cardiovascular disease, $[\ldots]$ to provide the best [...], physical, mental and social conditions, so that the
School of Pharmacy, Keele University, Staffordshire, ST5 5BG, UK

Tel +44I782 734772

Email s.j.white@keele.ac.uk 
patients may [...] resume optimal functioning in their community and through improved health behavior, slow or reverse progression of disease. ${ }^{2}$

Cardiac rehabilitation has been shown to benefit patients following myocardial infarction, as well as those who have had revascularization surgery (coronary artery bypass surgery or percutaneous coronary intervention) or implantable device insertion. ${ }^{3-5}$ Benefits have also been shown in patients with various other manifestations of CVD, including stable angina and heart failure..$^{3-5}$ Despite this, a significant proportion of eligible patients fail to receive $\mathrm{CR}$, including those ineligible for thrombolysis, socially deprived patients, women, ethnic minorities, and the elderly. ${ }^{3-5}$ Studies have consistently shown participation rates of less than $35 \%$ for patients who are eligible for CR across Europe, North America, and Australia, for example. ${ }^{3-10}$ This is partly due to variable CR service provision in terms of the nature and quality of services eg, there are wide differences in the duration of programs, type of exercise training, and other factors, ${ }^{4-9}$ although there are many other reasons why participation rates remain low. ${ }^{5,6}$ These broadly fall into three categories: reasons for eligible patients not being referred to CR; reasons why patients who are referred do not attend CR at all; and reasons why patients stop attending CR before completing the program. Subsequently, much effort in recent years has focused on attempting to improve attendance at $\mathrm{CR}$ and making $\mathrm{CR}$ available for all eligible patients. ${ }^{6-8}$ This has included development of standards and guidance about CR service provision, which details eligibility for $\mathrm{CR}$ and patient referral. . $^{2,6-8,11}$

These standards and guidance also specify the components of $\mathrm{CR}$ that should be provided. Those that are recognized as being essential in international and national guidance and standards include education about health behavior, lifestyle modification where necessary (especially in relation to smoking, diet, and physical exercise), medical risk factor management, use of cardioprotective medicines and implantable devices, and psychosocial health management. ${ }^{2}$ It is increasingly recognized that CR should be provided on a long-term basis and subject to regular audit and evaluation to ensure that service provision meets appropriate national standards. Amongst others, these standards include the British Association for Cardiovascular Prevention and Rehabilitation (BACPR) 2012 Standards and Core Components for Cardiovascular Disease Prevention and Rehabilitation, ${ }^{2}$ the American guidelines for CR and secondary prevention programs, ${ }^{8}$ and the European guidelines for CVD prevention. ${ }^{11}$
This paper has drawn on these standards and guidance, as well as reports and studies written in the English language from authoritative national and international institutions and organizations for the purpose of reviewing the impact of graded exercise undertaken as part of a CR program in the recovery period following a myocardial infarction. A pragmatic approach has been taken toward the breadth and complexity of the topics discussed, in that broad principles are discussed, with examples provided or references cited to illustrate the application of these principles in CR. This begins with a discussion of how CR may be best provided, which includes structuring exercises, tailoring CR, especially exercise, to individual patient needs, dominant themes in the largely qualitative body of literature on patient perspectives on lifestyle modification, and how health professionals can take a theoretically informed approach to changing patients' health behavior. The paper then turns to discussing the evidence-base relating to exercise-based $\mathrm{CR}$.

\section{How cardiac rehabilitation may be best provided}

Referral for CR should happen as soon as possible after a diagnosis of CVD has been made so that an initial assessment can be made of the patient's individual physical, psychological, and social needs, and a therapeutic plan agreed. ${ }^{2,7}$ Adoption of a menu-based approach to the interventions provided allows the CR program to be tailored to the patient's individual needs. ${ }^{2,7}$ Core interventions include structured exercise sessions, psychosocial support, and provision of information on various issues, including lifestyle recommendations and the use, benefit, and harms of medicines. ${ }^{2,3}$ Providing these interventions typically involves multidisciplinary input from health professionals, such as nurses, physiotherapists and other exercise professionals, occupational therapists, cardiologists, dietitians, pharmacists, and social workers.

\section{Structuring exercises}

Structured exercises are recommended for most patients, but the timing and location is largely dependent on individual patient needs and circumstances. For some patients, especially especially those who have had an ST-elevation myocardial infarction, undertaking structured exercise sessions occurs after a period of convalescence following discharge from hospital where as far as possible they gradually resume activities of daily living. The exercise sessions may be undertaken as part of a group-based rehabilitation program provided in a hospital or a community venue, or may be home-based according to an individualized physical activity plan or a 
validated program such as the Heart Manual ${ }^{12}$ or the Road to Recovery. ${ }^{13}$ Home-based programs may be undertaken as well as or instead of group-based sessions. ${ }^{7}$ Ideally, patients should be able to choose, given that home-based CR appears to be as effective as CR provided in a hospital or community setting, ${ }^{7,14}$ although in reality some patients may only be able to receive a home-based program because of difficulties accessing center-based programs. ${ }^{7}$ This may be more likely for patients with lower functional capacity or those who are vulnerable and at higher risk. ${ }^{7}$

Best practice standards and guidelines exist for physical activity and prescription of exercise in $\mathrm{CR}$, examples of which include the Association of Chartered Physiotherapists in Cardiac Rehabilitation (ACPICR) 2009 standards for physical activity and exercise in the cardiac population. Among other things, these standards specify service agreements that should be in place for referral of patients for the exercise component of CR, staffing for group-based exercise sessions in hospital or community settings, appropriate monitoring of patients undertaking exercise, the emergency protocols that should be in place, the emergency equipment that should be available, and the documentation that should be maintained. Exercise professionals who lead the exercise component of CR programs should be appropriately qualified and competent according to, for example, the ACPICR 2008 Competencies for the Exercise Component of Phase III Cardiac Rehabilitation. ${ }^{15}$

The standards also require that all patients receive an initial assessment prior to undertaking the exercise component, which as a minimum should include assessment of the following: 2,7

- physical measurements of heart rate, blood pressure, blood glucose, blood lipids, weight, body mass index, waist to hip ratio, and waist circumference;

- results of investigations that could indicate limitations in performing physical activity (eg, electrocardiographic exercise tolerance test);

- current and previous physical activity, including the individual's goals for physical activity and exercise, and their informed consent to take part in the structured exercises;

- comorbidities, signs and symptoms, and medicines taken (the standards specify certain comorbidities that prohibit exercise, eg, as detailed in the ACPICR standards ${ }^{7}$ ).

Patients should be risk-stratified on the basis of the initial assessment as low, moderate, or high risk. ${ }^{2,7}$ Factors that indicate high risk include, among others, a history of cardiac arrest, complex arrhythmias, onset of angina or other symptoms at low-level exertion or during recovery, and chronic heart failure or impaired cardiac output (ie, left ventricular ejection fraction less than $40 \%){ }^{7,8}$ This stratification will determine the patient's individual exercise prescription and the level of supervision needed when undertaking exercises, guidance and support required on activities of daily living, and the most appropriate location for CR (eg, hospital, community, or home). As part of the initial assessment, patients should also undergo a functional capacity test, performed according to standardized procedures, which should include calculation of the patient's exercise target heart rate (for example, using the Karvonen formula ${ }^{16}$ ). All patients should receive education about preparing for physical activity (eg, being suitably dressed and making sure essential medicines are available, such as glyceryl trinitrate), how to exercise safely (eg, the need for warm up and cool down periods, and recognizing symptoms during exercise) and when to stop or seek advice (eg, because of the onset of signs or symptoms of over-exertion). ${ }^{7,8}$

In addition to at least 30 minutes worth of daily physical activity, exercise sessions to improve cardiovascular fitness should be undertaken a minimum of 2-3 times per week. ${ }^{7,17}$ These may be combined with resistance training, or resistance training may be undertaken independently two or three times per week. ${ }^{7}$ The prescription of resistance training is usually based on the maximum weight that can be used to complete one repetition and the usual prescription involves performing $30 \%-40 \%$ of one repetition for upper body exercises and $40 \%-60 \%$ of one repetition for lower body exercises. ${ }^{7}$ Each set of exercises includes 10-15 repetitions and usually 8-10 sets of different exercises are performed. ${ }^{7}$

Exercise sessions, whether in a home, community, or hospital setting, should be designed to vary the frequency, intensity, duration, and type of exercise. All exercise sessions must include an initial warm-up period, before a conditioning period, and finish with a cool-down period. ${ }^{7,8}$ The warm-up period should be low-intensity, graduated, and last approximately 15 minutes (less for patients with lower physical function). It should involve pulse-raising activities and static stretching, and should ensure that joints are mobilized and relevant large muscle groups are warmed up. The conditioning period usually involves moderate-intensity aerobic exercises (ie, $50 \%-70 \%$ of heart rate reserve or $60 \%-75 \%$ of maximum heart rate ${ }^{7,8}$ ) designed to suit a range of fitness levels. Examples of common exercises include walking, cycling, circuit training, or use of exercise machines, such as treadmills or exercise cycles. This period should last for 20-30 minutes, although patients may have to gradually 
build up to being able to exercise continuously for this long. The cool-down period should last 10-15 minutes and should involve a gradual decrease in activity (eg, the warm-up period in reverse). This has been shown to reduce the incidence of hypotension, ischemic events, and arrhythmias, although given the increased risk of these adverse effects in the first 30 minutes after an exercise session, patients should be supervised for at least 15 minutes from the end of the cooldown period. ${ }^{7}$

The ACPICR also recommends that patients should maintain a good posture throughout exercise sessions, that the exercises should ensure muscle balance and that the target muscle groups should be varied to avoid overloading or overusing any particular group. ${ }^{7}$ To avoid adverse cardiovascular effects, patients should be advised to avoid changing position too quickly because this can cause hypotension and certain exercises should be avoided or modified. Exercises that can cause a sudden rise in blood pressure should be avoided (eg, those that involve breath holding), whilst exercises that may decrease cardiac output should be modified by adjusting the distribution of the power output between the upper and lower body (eg, arm exercise performed above chest height when sitting down should be combined with low-intensity leg work, such as alternate heel raises).

Patients undertaking group-based exercise sessions in hospital or community settings need to be screened prior to each session to ensure that it is safe for them to exercise and monitored during sessions to ensure that the exercise is safe and effective for them (alternative arrangements apply to home-based programs). Regular assessments of patients' progress need to be made, with adjustments made to the exercise prescription where appropriate to help them achieve their individual goals. Screening prior to sessions should focus on assessing any changes to the patient's health or medication since the previous assessment, assessing the details from other health professionals' reviews or results from investigations, and assessing the patient's response to recent physical activity. Monitoring during exercise sessions should include measurement of heart rate and blood pressure, completion of exertion rating scales and observation of the patient for signs or symptoms of over-exertion (eg, breathlessness, chest pain, dizziness, excessive fatigue or sweating, or a poor color), in addition to ensuring that their exercise technique and posture is correct. ${ }^{7}$

In addition, patients should be taught to self-monitor so that they can exercise safely on their own. This is particularly important for home-based exercise programs. Patients need to learn when it is safe to exercise (in a similar way to screening prior to exercise sessions) and to be aware of the signs and symptoms of over-exertion when exercising, as well as the appropriate action to take if they do over-exert themselves (eg, stop exercising, reduce the intensity, or take medication to relieve symptoms, such as glyceryl trinitrate for chest pain). Using rating scales for physical exertion such as the Borg scale, ${ }^{18}$ patients can learn to correlate their feelings of exertion (eg, in terms of muscle fatigue and breathlessness) with objective measures of heart rate. This allows patients to self-monitor accurately and regulate their exertion to reach their optimum level of exercise intensity during exercise sessions safely, which is the goal for longterm independent care.

\section{Tailoring to individual patient's needs}

As has already been pointed out, CR service provision should be tailored to patients' individual needs. To do this, an initial assessment of the patient's needs should be made by an appropriately qualified member of the CR team, and the services and interventions to be provided should be discussed and agreed with the patient. ${ }^{2,8}$ This should take account of factors such as the patient's age, gender, disabilities, socioeconomic status, and ethnicity or cultural background, including religious beliefs. In relation to physical exercise, a specific exercise prescription needs to be developed with the patient by an appropriately qualified exercise professional, following assessment of the patient's functional capacity and stratification of their risk as low, moderate, or high., ${ }^{2,7}$ The findings of a recent nonsystematic literature review suggest that this could include high-intensity interval training, especially in the most deconditioned patients and specifically if passive recovery periods are incorporated. ${ }^{19}$ Assessment of the level of supervision that the patient requires when exercising will take account of whether they have problems with hearing, sight, or balance, or have any other musculoskeletal or neurological problems.

There are additional considerations that will apply to highrisk patients with heart failure or implantable devices, and those who have had cardiac transplants. These are detailed in the standards and guidance, and include additional issues that should be assessed, limitations on the exercise prescription, types of exercise recommended, and particular things to avoid. ${ }^{7}$ Examples of these considerations include heart failure patients being at high risk of developing ischemia, hypotension, and arrhythmias, especially in the period after an exercise session, so short but frequent sessions are recommended initially, with warm-up and cool-down periods of the same duration as the conditioning period. ${ }^{7}$ Lower exercise 
intensities are recommended for patients having implantable cardioverter defibrillators with monitoring to ensure that the patient's heart rate remains below the implantable cardioverter defibrillator detection threshold. ${ }^{20}$ Hypotension due to low pump flow is common in patients with left ventricular assist devices, and the patient or their carer must know how to respond appropriately if this occurs. ${ }^{7}$ Increases and decreases in heart rate in response to starting or stopping exercise are delayed in cardiac transplantation patients due to denervation of the heart during surgery, which means that prolonged warm-up and cool-down periods are needed. ${ }^{21}$

However, whilst these measures should ensure that CR service provision is tailored to the patient's needs, there are a variety of other factors that may impinge on the likelihood of patients engaging with CR services or making and maintaining modifications to their lifestyle in particular. With this in mind, the discussion here now turns to considering patient perspectives on lifestyle modification before discussing potential approaches that health professionals in CR can adopt to encourage health behavior change.

\section{Patient perspectives on lifestyle modification}

Many studies of post-myocardial infarction patients describe the process of recovery as having a number of components, for example, Johnson and Morse's four-stage model of regaining a sense of control after myocardial infarction. ${ }^{22}$ Their first stage is "defending oneself" against a threatened loss of control, which broadly corresponds to the process of making the decision to seek medical help for the symptoms of myocardial infarction. The second stage is "coming to terms" with having had a myocardial infarction, and this includes looking for causes.

Commonly perceived causes include stress, heredity, and aspects of lifestyle, such as smoking, poor diet, and lack of exercise. ${ }^{22}$ Patients may feel angry that they have had a myocardial infarction, particularly if they had followed health recommendations, ${ }^{23}$ they may feel guilt and self-reproach because of their lifestyle prior to their myocardial infarction, ${ }^{24}$ or they may feel that other people blame them..$^{25}$ Other components of the second stage of recovery are termed "facing limitations" and "considering the implications of the heart attack on the future". A lot of studies of post-myocardial infarction patients have noted patients' residual fear and anxiety, especially of having another (fatal) CVD-related event.

The third stage is "learning to live" where patients negotiate an uncertain process of adjustment and regaining trust in their abilities. A crucial step in this process is "preserving a sense of self", since Johnson and Morse noted that many patients felt that physical limitations reduced their sense of self-worth. ${ }^{22}$ Many other studies have reported that patients often have physical limitations, most commonly tiredness, chest pain, or breathlessness, and subsequently feel frustrated, depressed, angry, tearful, and easily irritated. ${ }^{23-27}$ Fear of having another CVD-related event and the associated anxiety may cause breathlessness and increases in blood pressure and heart rate. ${ }^{28}$ Breathlessness and chest pain may cause fear, to the point where patients may be afraid to exert themselves at all. ${ }^{23}$ On the other hand, patients may be concerned that others might see them as weak or incapable, and so attempt to "put the record straight" by exerting themselves more than recommended..$^{22}$ This third stage of recovery also includes "establishing guidelines for living", where patients test limitations, learn to read their bodies, and modify their lifestyles. The fourth stage of recovery is "living again", which is the point where, without forgetting that they have had a myocardial infarction, patients are able to put it behind them and allow other areas of their life to take precedence. ${ }^{22}$ This may involve long-term maintenance of the lifestyle modifications made.

Undertaking group-based or home-based physical exercise sessions is most likely to start when patients are at the third stage of their recovery, and studies have found ways in which CR may help patients but also barriers to accessing it. Clark et al found that patients who attended CR programs often reported increased trust in their bodies, greater knowledge of their physical limits, and a heightened sense of fitness. ${ }^{29,30}$ They noted that patients benefited from group camaraderie and the opportunity to compare their progress with that of others, as has been found by other studies. ${ }^{31,32}$ A number of barriers to patients attending CR have been reported, of which transport difficulties have been commonly cited. ${ }^{33-39}$ Several studies found that a number of patients felt embarrassed about exercising in public, ${ }^{29,34,39}$ although this may lessen through attendance at CR ${ }^{29}$ Other barriers included limited resources restricting CR service capacity $^{38,39}$ or quality. ${ }^{37,40}$ Waiting lists of up to 12 months in some cases have been reported and patients were often unable to access information about the availability of CR, especially those patients who did not speak English or who used sign language. ${ }^{39}$

In relation to lifestyle modification, it is well established in the literature that patients who have had a myocardial infarction often experience difficulty in making and maintaining lifestyle changes. ${ }^{41-45}$ The literature also 
establishes that some patients, but typically not all, make and maintain some lifestyle changes but not necessarily all of the changes recommended..$^{23,24,27,41,44,46}$ In relation to physical exercise, studies have found that patients may find maintaining regular physical exercise difficult because of a dislike of exercise, poor weather, or experiencing symptoms. ${ }^{47}$ Patients may perceive the recommendations to be unrealistic and "asking too much", ${ }^{44}$ or report a lack of access to facilities for exercise. ${ }^{45}$ Cultural barriers have also been reported, such as modesty issues in mixed-gender exercise facilities or a lack of women-only exercise facilities. ${ }^{48}$ Studies have found that some patients perceive there to be too many changes to make at once, and as a result, patients may overcompensate in one area of lifestyle modification to make up for failing to modify another. ${ }^{43,44}$ Some studies have noted gender differences in the lifestyle modifications made, for example, that women tended to view exercise primarily in terms of losing weight and keeping fit and mobile, rather than in terms of CVD prevention. ${ }^{48,49}$ Studies have reported that women may perceive that the activity inherent in their domestic lives provides sufficient moderate physical activity and avoids potential harm from over-exercising. ${ }^{48,50}$

Patients may adopt an approach to lifestyle modification that follows from their own perspectives on their risk of experiencing further CVD-related events, such that the aspects of lifestyle that they modify and maintain are those that are perceived to have been likely causes of their myocardial infarction. ${ }^{22,24,27,46,51}$ Conversely, aspects of lifestyle that are not perceived as likely causes may not be changed, or are initially changed but then not maintained. As such, patients may view lifestyle modification in terms of reducing the risk of experiencing further CVD-related events, ${ }^{24,47}$ or they may view lifestyle modification in terms of leading to improvements in their health or achieving recovery. ${ }^{22,27,41,46}$ Indeed, studies have reported that some patients who initially viewed lifestyle modification in terms of getting "back to normal" over time came to see lifestyle modifications as long-term preventative measures because they were "vulnerable" to myocardial infarction, but then had low motivation to maintain lifestyle modifications because they had "got back to normal". ${ }^{27,46}$

This suggests that CR patients may not view lifestylerelated risk reduction in terms of a series of targets that may require lifestyle modification to achieve, as is advocated in national standards and guidance. ${ }^{2,3,52}$ Neither do CR patients appear to accept uncritically the generalized advice about lifestyle changes that they are given on CR programs. ${ }^{44}$ Rather, patients seem to relate information about reduction of lifestyle risk factors to their own circumstances, which highlights the tension between approaches to reduction of CVD risk in the population at large and individual patient perspectives. $^{44,53}$

\section{Changing health behaviors}

This tension has in part arisen from application of population-based estimates of risk to individual people, in order to reduce the burden of CVD in the whole population. ${ }^{53}$ Patients with established CVD (eg, those who have had a myocardial infarction) are considered to be at high risk (as assessed by algorithms ${ }^{54}$ ), and in line with evidence-based clinical guidelines, ${ }^{52}$ are subsequently advised to reduce lifestyle risk factors (ie, meet a series of lifestyle targets), which for many patients requires lifestyle modification to achieve. The difficulty with this approach is that assessments of any person's individual risk remain based on population data, irrespective of the sophistication of the technique. It cannot be predicted with any certainty which individuals will be affected by CVD, or which patients with established CVD will experience further CVD-related events. As such, there is no guarantee that further events will be prevented if lifestyle targets are met; neither does failure to meet lifestyle targets guarantee that CVD-related events will occur.

Nevertheless, there continues to be a strong emphasis in health promotion and health education strategies and activities, such as CR, on heightening peoples' awareness of "their" risk of cardiovascular disease and on their personal responsibility for reducing lifestyle risk factors. This makes the all-important step of personalizing risk such that it moves from the population as a whole to the individual, and "choosing" a healthy lifestyle is promoted as a rational response to awareness of health risks. ${ }^{53}$ This ideology of risk avoidance being rational behavior and risk-taking being irrational behavior has considerable resonance with certain psychological models of changing health behavior, such as the health belief model, ${ }^{55}$ among others. According to this model, people will take appropriate preventative action if they view themselves as being at significant risk of illness where there would be serious consequences and that taking preventative measures would be effective without the costs outweighing the benefits. This tends to assume a direct relationship between taking appropriate preventative measures and having adequate "knowledge" about the risk, and views 
failure to take preventative action as being either due to ignorance or irrationality, for example, fatalistic "misconceptions" about CVD-related risk. ${ }^{56,57}$

There is clearly a role for provision of detailed information about specific lifestyle modifications and there is evidence of short-term morbidity and cost-effectiveness benefits from therapeutic patient education programs. ${ }^{58,59}$ However, patients may well decide for themselves how relevant this information is to their own circumstances, which does not necessarily involve following lifestyle recommendations. ${ }^{44,51}$ Given the arguments presented above, this appears to be neither irrational nor ignorant from the patient's point of view. Indeed, as is well established in the literature, people's choice not to follow lifestyle recommendations (whether they have got CVD or not) is rarely because they are unaware that these aspects of lifestyle may constitute a health risk. ${ }^{56}$

Health professionals should bear these points in mind when approaching health behavior change with CR patients but, this said, models of health behavior change can be useful when planning interventions with patients to motivate and support them to make and maintain lifestyle changes. ${ }^{59}$ Models such as the theory of planned behavior ${ }^{60}$ that take account of patients' self-efficacy (their assessment of whether they are able to change successfully) and their subjective norms (beliefs about what other people think they should do and their desire to comply with these wishes) have been shown to be effective in achieving behavior change. ${ }^{61}$ The transtheoretical model ${ }^{62}$ also incorporates elements of self-efficacy and appears to be popular in CR. ${ }^{7}$ One of the core constructs of this model is a series of five stages of behavior change, ie, precontemplation, contemplation, preparation, action, and maintenance. This is based on the assumption that behavior change is an ongoing process and that people have varying levels of motivation or readiness to change. Particular barriers to physical activity that have been identified in patients with cardiovascular disease include comorbidities, age, exercise tolerance, anxiety and depression, and social and ethnic origin. ${ }^{63}$ People may move backwards or forwards through these stages and may require several cycles before successful change is established, such that the patient feels no desire or temptation to return to their previous behavior. As such, relapse is considered to be a normal aspect of the process. A key benefit of the transtheoretical model is that it provides a useful way of tailoring interventions to the stage that patients are at, although concerns remain that health inequalities may be exacerbated by categorizing people as precontemplative, because these patients are likely to be those who are already most disadvantaged. ${ }^{64}$

\section{Evidence for morbidity and mortality benefits}

There is recent high-profile systematic review evidence for exercise-based CR having mortality and morbidity benefits. ${ }^{1,65}$ This supports the findings from a number of older systematic reviews of an all-cause mortality reduction of approximately $13 \%$ and a reduction in cardiac mortality in the region of $26 \% .{ }^{66,67}$ The evidence also suggests that exercise-based CR leads to a reduction in reinfarction post myocardial infarction and in unplanned readmissions to hospital. ${ }^{68-70}$ These findings indicate that exercise-based CR is a cost-effective intervention in the management of CVD. ${ }^{52}$

A 2011 Cochrane review found that exercise-based $\mathrm{CR}$ for coronary heart disease effectively and significantly reduces medium-term to long-term (ie, $\geq 12$ months) all-cause and cardiovascular mortality, and short-term (ie, <12 months) hospital admissions. ${ }^{1}$ However, it was not found to be effective in reducing the total number of myocardial infarctions or revascularization procedures (percutaneous transluminal coronary angioplasty and coronary artery bypass surgery). Exercise-based CR was also found to increase health-related quality of life significantly compared with usual care (ie, that did not include structured exercise or advice about exercise) in seven of the 10 studies that measured this using a validated instrument. The systematic review included 47 randomized controlled trials (RCTs) that between them had randomized 10,794 patients to either exercise-based CR or usual care and had followed patients up for at least 6 months afterwards. The term "exercise-based CR" included any sort of structured exercises in a hospital, community, or home setting, with or without educational and psychosocial interventions. Participants included in these studies had had a myocardial infarction or revascularization surgery (coronary artery bypass surgery, percutaneous transluminal coronary angioplasty, or coronary artery stenting) but were excluded if they had heart failure or an implantable device fitted (ie, for cardiac resynchronization or defibrillation). It is worthy of note that 30 of the 47 RCTs included only post-myocardial infarction patients, and in all RCTs, middle-aged males were somewhat overrepresented in the participant populations.

Similar mortality and morbidity results were found in a 2011 systematic review of 34 RCTs with a total of 6,111 participants that assessed the effects of exercise-based CR for post-myocardial infarction patients compared with a 
control group that did not undertake exercise. ${ }^{65}$ This review found that the risk of cardiac mortality and all-cause mortality, as well as reinfarction post myocardial infarction was significantly lower for patients who had undertaken exercise-based CR than for those who had not undertaken a program of structured exercise. The other significant finding was that CR appeared to have positive effects on CVD risk factors, both lifestyle-related (eg, smoking and body weight) and physiological (eg, blood pressure and blood lipids). The review included 19 RCTs of exercise-only CR, 14 RCTs of CR programs that included exercise and other interventions, and one RCT that used both types of CR in separate intervention arms. However, it is worth noting that the type of exercise that participants undertook in these CR programs varied too, given that in 20 RCTs, participants only did aerobic exercise, whilst seven RCTs combined aerobic exercise with anaerobic exercise, and in seven RCTs the type of exercise was not specified.

However, there has recently been much debate about the effect of exercise-based CR in light of the findings of RAMIT (the Rehabilitation After Myocardial Infarction Trial), which found no benefits in terms of mortality, psychological status, or health-related quality of life, and that CR participants were likely to be slightly less physically active at 12 months than the control group. ${ }^{71}$ This study involved 1813 patients who were randomized to either comprehensive CR (ie, exercise and other interventions, including those of a psychosocial and educational nature) or "usual care". Much of the subsequent debate about the implications of these findings has focused on the relevance of evidence for the efficacy of CR and the effectiveness of CR services that are actually provided for CVD patients.

In terms of efficacy, the RAMIT investigators question the view that $\mathrm{CR}$ reduces mortality by as much as $20 \%$ on the basis that the RCTs included in systematic reviews were mostly small-scale trials with findings that have become outdated by substantial changes in the management of CVD. ${ }^{71}$ This argument is not without foundation, given that of the 47 studies included in the 2011 Cochrane review, 33 were published before the year 2000 and only three of the studies included more than 300 participants, 17 studies included fewer than 100 participants, and 16 studies included 100-200 participants. ${ }^{1}$ However, substantial mortality benefits have also been found in recent studies, including large-scale studies that have involved over 70,000 patients. $^{72}$ Indeed, the Cochrane review authors have since reported a reduction in all-cause mortality of $11 \%$ (down from the $13 \%$ previously reported) compared with "usual care" after adding the
RAMIT findings into their analyses. ${ }^{73}$ As such, the totality of the evidence continues to demonstrate mortality benefits of exercise-based CR, ${ }^{74}$ despite the dispute about the magnitude of that benefit.

The debate relating to the effectiveness of CR services provided principally concerns differences between the protocols for CR provision in RCTs and CR service provision in "real life". The CR programs that participants in the RAMIT study undertook complied with guidance issued in 1995 by the then British Association for Cardiac Rehabilitation (now BACPR), but were nonetheless "real life" CR programs. ${ }^{71}$ Therefore, the study findings could be interpreted as being an indictment on actual service provision rather than best possible service provision. ${ }^{74}$ Seen from this perspective, the RAMIT study findings appear somewhat less controversial, given that it is well established that there is considerable variation in the quality and nature of CR service provision in the UK and many other countries. ${ }^{3-5}$ Resolving this problem is crucial if patients are to benefit fully from graded exercise undertaken as part of a CR program in the recovery period following a myocardial infarction.

\section{Conclusion}

The components of CR recognized as essential in international and national guidance and standards include education about health behavior, lifestyle modification where necessary (especially in relation to smoking, diet, and physical exercise), medical risk factor management, use of cardioprotective medicines and implantable devices, and psychosocial health management. The totality of the evidence, which includes recent systematic reviews, continues to demonstrate mortality and morbidity benefits of exercise-based CR, despite debate about the magnitude of benefit. However, wide variance in the quality and nature of CR service provision means that there is no guarantee that patients will fully benefit.

CR should be tailored to the individual patient's needs, but structured exercise is recommended for most patients. Following initial assessment, the individual patient's exercise prescription and the supervision and support they need should be determined. This usually involves completing at least 30 minutes of daily physical activity and exercise sessions two or three times per week. Exercise sessions, whether based in hospital, the community or at home, should be designed to vary the frequency, intensity, duration, and type of exercise. They must include a warm-up period, a conditioning period, and a cool-down period. Patients should be taught to self-monitor so that they can exercise safely on their own. Regular assessments of patients' progress need to be 
made, with adjustments made to the exercise prescription as appropriate. In designing interventions to support patients to change health behavior, health professionals should recognize that patients may only modify aspects of lifestyle perceived as causes of their CVD and so, for example, may not do the recommended amount of exercise if they do not perceive lack of exercise to be a cause of their CVD.

\section{Disclosure}

The author reports no conflicts of interest in this work.

\section{References}

1. Heran B, Chen J, Ebrahim S, et al. Exercise-based cardiac rehabilitation for coronary heart disease. Cochrane Database Syst Rev. 2011;7:CD001800.

2. British Association for Cardiovascular Prevention and Rehabilitation. The BACPR Standards and Core Components for Cardiovascular Disease Prevention and Rehabilitation 2012. 2nd ed. London, UK: British Association for Cardiovascular Prevention and Rehabilitation; 2012.

3. Department of Health. National Service Framework for Coronary Heart Disease. London, UK: Department of Health; 2000.

4. Williams M, Ades P, Hamm L, Keteyian S. Clinical evidence for a health benefit from cardiac rehabilitation: an update. Am Heart $J$. 2006;152:835-841.

5. Bethell H, Lewin R, Dalal H. Cardiac rehabilitation in the United Kingdom. Heart. 2009;95:271-275.

6. NHS Improvement. Quality, innovation and value in cardiac rehabilitation: commissioning for improvement. London, UK: Department of Health; 2012. Available from: http://www.improvement.nhs.uk/ePublications/cardiacrehab/. Accessed July 2, 2013.

7. Association of Chartered Physiotherapists in Cardiac Rehabilitation. ACPICR Standards for Physical Activity and Exercise in the Cardiac Population 2009. London: Association of Chartered Physiotherapists in Cardiac Rehabilitation; 2009. Available from: http://www.acpicr.com/ sites/default/files/Acpicr\%20standards_1.pdf. Accessed July 1, 2013.

8. Balady G, Williams M, Ades P, et al. Core components of cardiac rehabilitation/secondary prevention programs: 2007 update: a scientific statement from the American Heart Association Exercise, Cardiac Rehabilitation, and Prevention Committee, the Council on Clinical Cardiology; the Councils on Cardiovascular Nursing, Epidemiology and Prevention, and Nutrition, Physical Activity, and Metabolism; and the American Association of Cardiovascular and Pulmonary Rehabilitation. Circulation. 2007;115:2675-2682.

9. Bjarnason-Wehrens B, McGee H, Zwisler A, et al. Cardiac rehabilitation in Europe: results from the European Cardiac Rehabilitation Inventory Survey. Eur J Cardiovasc Prev Rehabil. 2010;17:410-418.

10. Bunker S, McBurney H, Cox H, Jelinek M. Identifying participation rates at outpatient cardiac rehabilitation programs in Victoria, Australia. J Cardiopulm Rehabil. 1999:334-338.

11. Perk J, DeBacker G, Gohlke H, et al. European Guidelines on cardiovascular disease prevention in clinical practice (2012). The Fifth Joint Task Force of the European Society of Cardiology and other societies on cardiovascular disease prevention in clinical practice (constitutes by representatives of nine societies and invited experts). Eur J Prev Cardiol. 2012;19:585-667.

12. National Health Service. Heart Manual: A Self-Management Programme for Patients With Coronary Artery Disease. Edinburgh, Scotland: NHS Lothian; 2009. Available from: http://www.theheartmanual.com/About/ Documents/Leaflet.pdf. Accessed June 18, 2013.

13. British Heart Foundation. Road to Recovery: A Cardiac Rehabilitation Programme. London, UK: British Heart Foundation; 2007.
14. Dalal H, Zawada A, Jolly K, Moxham T, Taylor R. Home based versus centre based cardiac rehabilitation: cochrane systematic review and meta-analysis. BMJ. 2010;340:b5631.

15. Association of Chartered Physiotherapists in Cardiac Rehabilitation. ACPICR Competencies for the Exercise Component of Phase III Cardiac Rehabilitation 2008. London, UK: Association of Chartered Physiotherapists in Cardiac Rehabilitation; 2008.

16. Karvonen M. Problems of training of the cardiovascular system. Ergonomics. 1999;2:207-215.

17. Smith S, Allen J, Blair S, et al. AHA/ACC Guidelines for secondary prevention for patients with coronary and other atherosclerotic vascular disease: 2006 update: endorsed by the National Heart, Lung and Blood Institute. Circulation. 2006;113:2363-2372.

18. Borg G. Borg's Rating of Perceived Exertion and Pain Scales. Champaign, IL: Human Kinetics; 1998.

19. Guiraud T, Nigam A, Gremeaux V, Meyer P, Juneau M, Bosquet L. High-intensity interval training in cardiac rehabilitation. Sports Med. 2012;42:587-605.

20. Fitchet A, Doherty P, Bundy C, Bell W, Fitzpatrick A, Garratt C. Comprehensive cardiac rehabilitation programme for implantable cardioverter-defibrillator patients: a randomised controlled trial. Heart. 2003;89:155-160.

21. Haykowsky M, Eves N, Figgures L, et al. Effect of exercise training on $\mathrm{VO}^{2}$ peak and left ventricular function in recent cardiac transplant recipients. Am J Cardiol. 2005;95:1002-1004.

22. Johnson J, Morse J. Regaining control: the process of adjustment after myocardial infarction. Heart Lung. 1990;19:126-135.

23. Roebuck A, Furze G, Thompson D. Health-related quality of life after myocardial infarction: an interview study. $J$ Adv Nurs. 2001;34:787-794.

24. Jensen B, Petersson K. The illness experience of patients after a first time myocardial infarction. Patient Educ Couns. 2003;51:123-131.

25. Sutherland B, Jensen L. Living with change: elderly women's perceptions of having a myocardial infarction. Qual Health Res. 2000;10:661-676.

26. Kristofferzon M, Lofmark R, Carlsson M. Myocardial infarction: gender differences in coping and social support. $J A d v$ Nurs. $2003 ; 44: 360-374$

27. Wiles R. Patient's perceptions of their heart attack and recovery: the influence of epidemiological "evidence" and personal experience. Soc Sci Med. 1998;46:1477-1486.

28. Fraser S, Rodgers W, Daub B. Psychosocial correlates of cardiovascular reactivity to anticipation of an exercise stress test prior to attending cardiac rehabilitation: a preliminary test. J Appl Biobehav Res. 2008; 13:20-41.

29. Clark A, Barbour R, White M, MacIntyre P. Promoting participation in cardiac rehabilitation: patient choices and experiences. $J A d v$ Nurs. 2004; $47: 5-14$

30. Clark A, Whelan H, Barbour R, MacIntyre P. A realist study of the mechanisms of cardiac rehabilitation. JAdv Nurs. 2005;52:362-371.

31. Murie J, Ross A, Lough M, Rich D. Exploring post-myocardial infarction patients' perceptions of patient-mediated interventions for the secondary prevention of coronary heart disease (SIGN Guideline 41). Qual Prim Care. 2006;14:77-83.

32. Wingham J, Dalal H, Sweeney K, Evans P. Listening to patients: choice in cardiac rehabilitation. Eur J Cardiovasc Nurs. 2006;5:289-294.

33. Caldwell P, Arthur H, Rideout E. Lives of rural women after myocardial infarction. Can J Nurs Res. 2005;37:54-67.

34. Cooper A, Jackson G, Weinman J, Horne R. A qualitative study investigating patients' beliefs about cardiac rehabilitation. Clin Rehabil. 2005;19:87-96.

35. Hird C, Upton C, Chesson R. "Getting back to normal": patients' understanding of cardiac rehabilitation. Physiotherapy. 2004;90: $125-131$.

36. McSweeney J, Crane P. An act of courage: women's decision-making processes regarding outpatient cardiac rehabilitation attendance. Rehabil Nurs. 2001;26:132-140. 
37. O'Driscoll J, Shave R, Cushion C. A National Health Service Hospital's cardiac rehabilitation programme: a qualitative analysis of provision. J Clin Nurs. 2007;16:1908-1918.

38. Paquet M, Bolduc N, Xhignesse M, Vanasse A. Re-engineering cardiac rehabilitation programmes: considering the patient's point of view. J Adv Nurs. 2005;51:567-576.

39. Tod A, Lacey E, McNeill F. "I'm still waiting...": barriers to accessing cardiac rehabilitation services. J Adv Nurs. 2002;40:421-431.

40. Day W, Batten L. Cardiac rehabilitation for women: one size does not fit all. Aust J Adv Nurs. 2006;24:21-26.

41. Bergman E, Bertero C. You can do it if you set your mind to it: a qualitative study of patients with coronary artery disease. JAdv Nurs. 2001;36:733-741.

42. Boutin-Foster C. Getting to the heart of social support: a qualitative analysis of the types of instrumental support that are most helpful in motivating cardiac risk factor modification. Heart Lung. 2005;34: 22-29.

43. Condon $\mathrm{C}$, McCarthy G. Lifestyle changes following acute myocardial infarction: patients' perspectives. Eur J Cardiovasc Nurs. 2006;5:37-44.

44. Gambling T. A qualitative study into the informational needs of coronary heart disease patients. Int J Health Promot Educ. 2003;41:68-76.

45. Karner A, Tingström P, Albrandt-Dahlgren M, Bergdahl B. Incentives for lifestyle changes in patients with coronary artery disease. $J A d v$ Nurs. 2005;1:261-275.

46. Wiles R, Kinmonth A. Patients' understandings of heart attack: implications for prevention of recurrence. Patient Educ Couns. 2001;44:161-169.

47. LaCharity L. The experiences of younger women with coronary artery disease. J Womens Health Gend Based Med. 1999;8:773-785.

48. Sriskantharajah J, Kai J. Promoting physical activity among South Asian women with coronary heart disease and diabetes: what might help? Fam Pract. 2007;24:71-76.

49. Ruston A, Clayton J. Coronary heart disease: women's assessment of risk - a qualitative study. Health Risk Soc. 2002;4:125-138.

50. Clayton J, Ruston A. Exercising for a healthy heart: a qualitative study of women's beliefs. Health Educ J. 2003;62:29-40.

51. White S, Bissell P, Anderson C. A qualitative study of patients' perspectives on cardiac rehabilitation, lifestyle change and taking medicines: implications for service development. J Health Serv Res Pol. 2010; 15 Suppl 2:47-53.

52. National Institute of Health and Clinical Excellence. Secondary Prevention in Primary and Secondary Care for Patients Following a Myocardial Infarction. London, UK: National Institute of Health and Clinical Excellence; 2007.

53. Davison C, Davey Smith G, Frankel S. Lay epidemiology and the prevention paradox: the implications of coronary candidacy for health education. Sociol Health Illn. 1991;13:1-19.

54. British Cardiac Society; British Hypertension Society; Diabetes UK; HEART UK; Primary Care Cardiovascular Society; Stroke Association. JBS 2: Joint British Societies' guidelines on prevention of cardiovascular disease in clinical practice. Heart. 2005;91 Suppl 5:v1-v52.

55. Becker M. The health belief model and personal health behaviour. Health Educ Monogr. 1974;2:324-473.

56. Lupton D. Risk. London, UK: Routledge; 1999.
57. Wheatley E. Disciplining bodies at risk: cardiac rehabilitation and the medicalisation of fitness. J Sport Soc Issues. 2005;29:198-221.

58. Labrunée M, Pathak A, Loscos M, Coudeyre E, Casillas J, Gremeaux V. Therapeutic education in cardiovascular diseases: state of the art and perspectives. Ann Phys Rehabil Med. 2012;55:322-341.

59. National Institute of Health and Clinical Excellence. Behaviour Change. London, UK: National Institute of Health and Clinical Excellence; 2007.

60. Azjen I, Fishbein M. Understanding Attitudes and Predicting Social Behaviour. Englewood Cliffs, NJ: Prentice-Hall; 1980.

61. Blanchard C, Courneya K, Rodgers W, et al. Is the theory of planned behavior a useful framework for understanding exercise adherence during phase II cardiac rehabilitation? J Cardiopulm Rehabil. 2003;23:29-39.

62. Prochaska J, DiClemente C. The Transtheoretical Approach: Crossing Traditional Boundaries of Therapy. Homewood, IL: Dow Jones Irwin; 1984.

63. Reid R, Morrin L, Pipe A, et al. Determinants of physical activity after hospitalization for coronary artery disease: the Tracking Exercise After Cardiac Hospitalization (TEACH) study. Eur J Cardiovasc Prev Rehabil. 2006;13:529-537.

64. Brug J, Oenema A, Ferreira I. Theory, evidence and intervention mapping to improve behavior nutrition and physical activity interventions. Int J Behav Nutr Phys Act. 2005;2:2.

65. Lawler P, Filion K, Eisenberg M. Efficacy of exercise-based cardiac rehabilitation post-myocardial infarction: a systematic review and meta-analysis of randomised controlled trials. Am Heart J. 2011;162:571-584.

66. Jolliffe J, Rees K, Taylor R, Thompson D, Oldridge N, Ebrahim S. Exercise-based rehabilitation for coronary artery disease. Cochrane Database Syst Rev. 2000;4:CD001800.

67. Taylor R, Brown A, Ebrahim S, et al. Exercise-based rehabilitation for patients with coronary heart disease: systematic review and meta-analysis of randomised controlled trials. Am J Med. 2004;116:682-697.

68. Clark A, Hartling L, Vandermeer B, McAlister F. Meta analysis: secondary prevention programs for patients with coronary artery disease. Ann Intern Med. 2005;143:659-672.

69. Lam G, Snow R, Shaffer L, La Londe M, Spencer K, Caulin-Glaser T. The effect of a comprehensive cardiac rehabilitation program on 60-day hospital readmissions after an acute myocardial infarction. J Am Coll Cardiol. 2011;57:597.

70. Davies E, Moxham T, Rees K, et al. Exercise training for systolic heart failure. Cochrane systematic review and meta-analysis. Eur J Heart Fail. 2010;12:706-715.

71. West R, Jones D, Henderson A. Rehabilitation after myocardial infarction trial (RAMIT): multi-centre randomised controlled trial of comprehensive cardiac rehabilitation in patients following acute myocardial infarction. Heart. 2012;98:637-644.

72. Suaya J, Stason W, Ades P, Normand S, Shepard D. Cardiac rehabilitation and survival in older coronary patients. J Am Coll Cardiol. 2009;54:25-33.

73. Taylor R. The RAMIT trial: its results in the context of 2012 Cochrane review. Heart. 2012;98:672-673.

74. Doherty P, Lewin R. The RAMIT trial, a pragmatic RCT of cardiac rehabilitation versus usual care: what does it tell us? Heart. 2012;98:605-606
Research Reports in Clinical Cardiology

\section{Publish your work in this journal}

Research Reports in Clinical Cardiology is an international, peerreviewed, open access journal publishing original research, reports, editorials, reviews and commentaries on all areas of cardiology in the clinic and laboratory. The manuscript management system is completely online and includes a very quick and fair peer-review system.

\section{Dovepress}

Visit http://www.dovepress.com/testimonials.php to read real quotes from published authors. 